\title{
Queratocono en edad pediátrica: características clínico-refractivas y evolución. Centro de Especialidades Médicas Fundación Donum, Cuenca, Ecuador, 2015-2018
}

\section{Keratoconus in pediatric age: clinical-refractive characteristics and evolution. Donum Foundation Medical Specialties Center. Cuenca. Ecuador. 2015-2018}

\author{
Eduardo Rojas-Álvarez* \\ Facultad de Ciencias Médicas, Universidad de Cuenca, Centro de Especialidades Médicas Fundación Donum, Cuenca, Ecuador
}

\begin{abstract}
Resumen
Objetivo: Describir las características clínico-refractivas de pacientes con queratocono $(K C)$ en edad pediátrica y su evoIución, en el Centro de Especialidades Médicas Fundación Donum, Cuenca, Ecuador. Método: Se realizó un estudio observacional, prospectivo y longitudinal en 94 pacientes en edad pediátrica (188 ojos) con diagnóstico de KC, diagnosticados en 2015 y seguidos hasta 2018, en el Centro de Especialidades Médicas Fundación Donum, Cuenca, Ecuador. Se obtuvo la media aritmética de la muestra de: queratometría media, paquimetría en el punto más delgado y cilindro refractivo, en dos momentos. Se empleó el análisis de varianza (ANOVA) de medidas repetidas para un factor con ajuste del intervalo de confianza por Bonferroni y valor p. Resultados: Se obtuvo un aumento estadísticamente significativo entre el valor promedio de queratometría media de la muestra en el momento del diagnóstico (49.6 D) y el valor promedio de queratometría media a los 3 años de seguimiento $(52.4 D)(p=0.02 ; \alpha=0.05)$; una disminución estadísticamente significativa entre la paquimetría promedio de la muestra en el momento del diagnóstico $(482 \mu \mathrm{m})$ y la paquimetría promedio de la muestra a los 3 años de seguimiento $(459 \mu \mathrm{m})(p=0.03 ; \alpha=0.05) ; y$ un incremento estadísticamente significativo entre el cilindro refractivo promedio de la muestra en el momento del diagnóstico $(4.6 \mathrm{D})$ y el cilindro refractivo promedio de la muestra a los 3 años de seguimiento $(5.1 D)(p=0.01 ; \alpha=0.05)$. Conclusiones: Las modificaciones de queratometría, cilindro refractivo y paquimetría sugieren progresión de la enfermedad en este grupo de edad, en un periodo de 3 años.
\end{abstract}

Palabras claves: Queratocono. Queratometría. Paquimetría. Cilindro refractivo. Agudeza visual.

\section{Abstract}

Objective: To describe the clinical-refractive characteristics of KC patients at pediatric age and their evolution, at the Donum Foundation Medical Center, Cuenca, Ecuador. Method: An observational, prospective and longitudinal study was carried out in 94 pediatric patients (188 eyes) diagnosed with KC, diagnosed in 2015 and followed up to 2018, in the Donum Foundation Medical Center, Cuenca, Ecuador. The arithmetic mean of the sample was obtained: mean keratometry, pachymetry at the

Correspondencia:

*Eduardo Rojas-Álvarez

Tarqui 13-56 y Pío Bravo

Fecha de recepción: 26-02-2019

Fecha de aceptación: 10-05-2019

E-mail: drerojasalvarez@gmail.com DOI: 10.24875/RMO.M19000082
Disponible en internet: 01-09-2019 Rev Mex Oftalmol. 2019;93(5):221-232

www.rmo.com.mx 0187-4519/@ 2019 Sociedad Mexicana de Oftalmología. Publicado por Permanyer México. Este es un artículo Open Access bajo la licencia CC BY-NC-ND (http://creativecommons.org/licenses/by-nc-nd/4.0/). 
thinnest point and refractive cylinder, at two moments. The analysis of variance (ANOVA) of repeated measures was used for a factor with confidence interval adjustment by Bonferroni and $p$ value. Results: $A$ statistically significant increase was obtained between the mean value of average keratometry of the sample (49.6 D), at the time of diagnosis, and the mean value of average keratometry at 3 years of follow-up (52.4 D). $(p=0.02 ; \alpha=0.05)$, a statistically significant decrease between the average pachymetry of the sample, at the time of diagnosis $(482 \mu \mathrm{m})$, and the average pachymetry of the sample, at 3 years of follow-up $(459 \mu \mathrm{m})(p=0.03 ; \alpha=0.05)$ A statistically significant increase was obtained between the average refractive cylinder of the sample, at the time of diagnosis (4.6 D), and the average refractive cylinder of the sample, at 3 years of follow-up (5.1 D) ( $p=0.01 ; \alpha=0.05)$. Conclusions: The modifications of keratometry, refractive cylinder and pachymetry suggest progression of the disease in this age group, in a period of 3 years.

Key words: Keratoconus. Keratometry. Pachymetry. Refractive cylinder. Visual acuity.

\section{Introducción}

En nuestra práctica diaria de la oftalmología nos encontramos casos de diversa índole que, en ocasiones, se llevan toda nuestra atención por su relevancia clínica e interesante evolución. Las ectasias, específicamente el queratocono $(\mathrm{KC})$, con presentación en edad pediátrica, constituyen un reto clínico-quirúrgico. Independientemente, de los adelantos tecnológicos acontecidos en las últimas décadas, el manejo de un paciente pediátrico con $\mathrm{KC}$ puede conducir a un camino largo de seguimiento, no exento de dudas, contradicciones, decisiones quirúrgicas a destiempo, entre otras cuestiones, que meritan toda nuestra atención.

El KC pediátrico demuestra varios problemas de manejo distintivos en comparación con el KC adulto con respecto a la dificultad para el diagnóstico por topografía corneal (cooperación, accesibilidad) y las modificaciones en los esquemas de tratamiento. Las principales preocupaciones comprenden la progresión acelerada de la enfermedad en el grupo de edad pediátrica y el manejo de las comorbilidades como la queratoconjuntivitis vernal'.

El KC es una enfermedad inflamatoria o cuasiinflamatoria, en la cual la córnea adopta una forma cónica debido al adelgazamiento y protrusión ${ }^{2}$. Se presenta en la pubertad y progresa hasta la tercera o cuarta décadas de la vida. La incidencia es de 1/2,000 casos en la población ${ }^{1,3}$. Clínicamente esta ectasia conduce a miopía y astigmatismo irregular ${ }^{1-4}$. La etiología aún no está bien esclarecida. Surge comúnmente de la combinación de factores genéticos y ambientales ${ }^{2-4}$.

La resistencia biomecánica de la córnea depende de los enlaces covalentes entre las moléculas de colágeno. En el KC, los queratocitos activados invaden la membrana de Bowman previamente alterada, con apoptosis posterior de estos, pérdida del estroma anterior, y se provoca un adelgazamiento progresivo corneal. En el $\mathrm{KC}$ la resistencia estromal disminuye en un $50 \%{ }^{3-5}$.
El momento del diagnóstico de la enfermedad continúa siendo el eje angular de la predicción evolutiva. Pueden llegar casos en diferentes estadios, desde KC aún subclínicos, hasta leucomas corneales cicatrizales por episodios de hidrops agudos que pasaron desapercibidos, incluso pueden concomitar estadios muy diferentes de la enfermedad en un mismo paciente, lo que resalta su carácter asimétrico.

Existen diferentes estudios en el mundo y en Latinoamérica, sobre las características clínicas y refractivas de esta enfermedad. Los estudios más actuales se centran en la efectividad del cross linking como alternativa de tratamiento en pacientes pediátricos con la enfermedad $6-8$.

En Ecuador, Abril ${ }^{9}$, que realizó topografía corneal a 32 pacientes con esta enfermedad en Quito y Santo Domingo, obtuvo valores paquimétricos y queratométricos. Mansfield ${ }^{10}$ obtiene un $0.75 \%$ de frecuencia de KC en un grupo de pacientes en la ciudad de Quito que se les realizó topografía corneal. En Cuenca, Reynoso ${ }^{11}$ obtiene una frecuencia de la enfermedad del $28.6 \%$ en pacientes que se les realizó topografía corneal.

Sin embargo, los estudios no van dirigidos a grupos de edades específicas en las que aparece la enfermedad. Investigaciones del KC en edad pediátrica posibilitarían su diagnóstico precoz, así como una mayor efectividad y optimización de los tratamientos efectuados, con el objetivo de mejorar, o al menos mantener, la agudeza visual de los pacientes y disminuir la posibilidad de un trasplante de córnea.

\section{Objetivos}

\section{General}

- Describir las características clínico-refractivas de pacientes con KC en edad pediátrica y su evolución, en la Fundación Donum, Cuenca, Ecuador. 


\section{Específicos}

- Caracterizar la muestra de estudio de acuerdo a sus características sociodemográficas.

- Distribuir la muestra de acuerdo a enfermedades sistémicas asociadas, signos y síntomas.

- Clasificar los pacientes según defecto refractivo, patrón topográfico y tipo de KC.

- Determinar las posibles modificaciones de queratometría, paquimetría y cilindro refractivo entre el momento del diagnóstico y a los 3 años del mismo.

\section{Método}

Se realizó un estudio observacional, prospectivo y longitudinal en 94 pacientes en edad pediátrica (188 ojos) con diagnóstico de KC, diagnosticados en 2015 y seguidos hasta 2018, en el Centro de Especialidades Médicas Fundación Donum, Cuenca, Ecuador.

\section{Criterios de selección de los casos}

\section{Criterios de inclusión}

- Edad menor a 18 años.

- Diagnóstico de KC (se consideró para el diagnóstico la presencia de criterios topográficos/tomográficos para la enfermedad por Pentacam y la presencia de alteraciones clínicas en la exploración: irregularidad en el reflejo corneal, signo de Munson y de Rizzuti, presencia de anillo de Fleischer, estrías de Vogt).

\section{Criterios de exclusión}

- Pacientes que no acudieron a las visitas durante los 3 años.

- Tutores que no otorgaron el consentimiento informado para la participación en el estudio.

\section{Variables del estudio}

- Sociodemográficas (edad, sexo, lugar de residencia).

- Enfermedades asociadas.

- Síntomas.

- Signos.

- Defectos refractivos.

- Cilindro refractivo.

- Patrón topográfico.

- Tipo de KC.

- Queratometría media.
- Paquimetría (punto más delgado)

- Agudeza visual (sin corrección y con corrección)

\section{Métodos de obtención de la información, análisis estadístico y aspectos éticos}

La información fue obtenida a partir de las historias clínicas de cada paciente (variables sociodemográficas, enfermedades asociadas, síntomas, signos y defecto refractivo, cilindro refractivo y agudeza visual). Los datos queratométricos, paquimétricos y topográficos se obtuvieron a partir de Pentacam. La información fue procesada en el programa SPSS, versión 22, utilizando métodos de estadística descriptiva e inferencial expresados en frecuencias absolutas y frecuencias relativas. Se obtuvo la media aritmética de la muestra de: queratometría media, paquimetría en el punto más delgado y cilindro refractivo, en dos momentos: en el momento del diagnóstico y a los 3 años de seguimiento. Se empleó el análisis de varianza (ANOVA) de medidas repetidas para un factor, con ajuste del intervalo de confianza por Bonferroni, para establecer las diferencias entre los dos momentos del estudio, y valor $p$. Los padres o tutores otorgaron su consentimiento para la participación en el estudio y la investigación fue aprobada en el Comité de Bioética de la institución. Se indicó tratamiento quirúrgico con crosslinking a los pacientes estudiados, resultados que forman parte de investigaciones futuras.

\section{Resultados}

Como se puede observar en la tabla 1 , en el estudio predominaron los pacientes en el grupo de edad de 14 a 16 años (29.8\%), seguido del grupo de 11 a 13 años (27.6\%) y de 17 a 18 años (23.4\%). Se obtuvo un predominio de pacientes del sexo masculino (60.6\%), y la mayoría procedentes de la ciudad de Cuenca (48.9\%), seguido de Azogues (18.1\%) y Cañar (9.6\%).

Como se muestra en la tabla 2, predominaron pacientes con presencia de queratoconjuntivitis alérgica $(45.1 \%)$, seguidos en orden de frecuencia por pacientes con alergia (23.2\%), dermatitis atópica $(11.0 \%)$ y otras enfermedades como asma bronquial (7.3\%), estrabismo $(4.9 \%)$, trauma corneal penetrante $(2.4 \%)$, así como pacientes sin enfermedades $(6.1 \%)$.

Asimismo, como se muestra en la tabla 3 predominaron pacientes cuyo síntoma motivo de consulta fue la disminución de la agudeza visual (68.1\%), seguido de pacientes con prurito (12.8\%) y pacientes con cambio frecuente de corrección (10.6\%) e intolerancia a los lentes de contacto (8.5\%). 
Tabla 1. Distribución de los pacientes de acuerdo a las variables sociodemográficas (edad, sexo, lugar de residencia). Centro de Especialidades Médicas Fundación Donum, Cuenca, Ecuador (2015-2018)

\begin{tabular}{|l|c|c|}
\hline Edad (años) & Frecuencia & Porcentaje \\
\hline $5-7$ & 6 & 6.4 \\
\hline $8-10$ & 12 & 12.8 \\
\hline $11-13$ & 26 & 27.6 \\
\hline $14-16$ & 28 & 29.8 \\
\hline $17-18$ & 22 & 23.4 \\
\hline Total & & \\
\hline Sexo & 94 & 100 \\
\hline Femenino & & \\
\hline Masculino & 37 & 39.4 \\
\hline Total & 57 & 60.6 \\
\hline Lugar de residencia & 94 & 100 \\
\hline Cuenca & & \\
\hline Azogues & & 48.9 \\
\hline Cañar & & 18.1 \\
\hline Guayaquil & 46 & 9.6 \\
\hline Machala & 17 & 7.4 \\
\hline Quito & 9 & 5.3 \\
\hline Loja & 7 & 3.2 \\
\hline Riobamba & 5 & 3.2 \\
\hline Otras & 3 & 3.2 \\
\hline Total & 3 & 1.1 \\
\hline Ful & 3 & 100 \\
\hline
\end{tabular}

Fuente: Historias clínicas.

Tabla 2. Distribución de los pacientes de acuerdo a las enfermedades asociadas. Centro de Especialidades Médicas Fundación Donum, Cuenca, Ecuador (2015-2018)

\begin{tabular}{|l|c|c|}
\hline Enfermedades asociadas & Frecuencia & Porcentaje \\
\hline Queratoconjuntivitis alérgica & 37 & 45.1 \\
\hline Alergia sistémica & 19 & 232 \\
\hline Dermatitis atópica & 9 & 11.0 \\
\hline Asma & 6 & 7.3 \\
\hline Sin enfermedades & 5 & 6.1 \\
\hline Estrabismo & 4 & 4.9 \\
\hline Trauma corneal penetrante & 2 & 2.4 \\
\hline
\end{tabular}

Fuente: Historias clínicas.

En la tabla 4 podemos observar que el $48.9 \%$ de los casos presentó signo de tijera al examen, seguido en orden de frecuencia por el $29.8 \%$ con nervios corneales prominentes y el $24.5 \%$ con presencia de signo de Munson, otros signos, como el anillo de Fleischer y estrías de Vogt, se obtuvieron con menor frecuencia.
Tabla 3. Distribución de los pacientes de acuerdo a los síntomas (motivo de consulta). Centro de Especialidades Médicas Fundación Donum, Cuenca, Ecuador (2015-2018)

\begin{tabular}{|l|c|c|}
\hline Síntomas & Frecuencia & Porcentaje \\
\hline $\begin{array}{l}\text { Disminución lenta y } \\
\text { progresiva de la visión }\end{array}$ & 64 & 68.1 \\
\hline $\begin{array}{l}\text { Prurito } \\
\text { Cambio frecuente de } \\
\text { corrección }\end{array}$ & 12 & 12.8 \\
\hline $\begin{array}{l}\text { Intolerancia a lentes de } \\
\text { contacto }\end{array}$ & 10 & 10.6 \\
\hline \begin{tabular}{l} 
Total \\
\hline
\end{tabular} & $\mathbf{9 4}$ & 8.5 \\
\hline
\end{tabular}

Fuente: Historias clínicas.

Tabla 4. Distribución de los pacientes de acuerdo a signos. Centro de especialidades médicas Fundación Donum, Cuenca, Ecuador (2015-2018)

\begin{tabular}{|l|c|c|}
\hline Signos & Frecuencia & Porcentaje \\
\hline Signo de tijera & 46 & 48.9 \\
\hline Nervios corneales prominentes & 28 & 29.8 \\
\hline Signo de Munson & 28 & 24.5 \\
\hline Anillo de Fleischer & 6 & 6.4 \\
\hline Estrías de Vogt & 6 & 6.4 \\
\hline Fuente: Historias clínicas. & & \\
\hline
\end{tabular}

La tabla 5 muestra que el defecto refractivo que predominó en el estudio es el astigmatismo miópico compuesto $(64.4 \%)$, seguido del astigmatismo mixto $(30.8 \%)$ y el astigmatismo miópico simple (4.8\%). No hubo pacientes con miopía pura o hipermetropía pura en la muestra estudiada.

En la tabla 6 podemos ver que el $35.1 \%$ de los ojos estudiados presentó un patrón topográfico de botón de camisa asimétrico con desviación de los ejes en J, seguido de patrón de botón de camisa asimétrico con encorvamiento inferior $(25.5 \%$ ) y patrón de astigmatismo irregular (22.3\%). El 17.1\% presentó patrón de botón de camisa asimétrico con encorvamiento superior (17.1\%).

La tabla 7 muestra un predominio del tipo 2 de KC (25.5\%), seguido del tipo $3(23.4 \%)$ y del tipo $1(19.2 \%)$. Con menor frecuencia se observaron los KC de tipo 5 (17.0\%) y tipo 4 (14.9\%).

La tabla 8 muestra un predominio de casos con queratometrías medias entre 45 y $49.9 \mathrm{D}$ en el momento del diagnóstico. El predominio de este grupo se mantuvo a 
Tabla 5. Distribución de los pacientes de acuerdo al defecto refractivo. Centro de Especialidades Médicas Fundación Donum, Cuenca, Ecuador (2015-2018)

\begin{tabular}{|l|c|c|}
\hline Defecto refractivo & Frecuencia & Porcentaje \\
\hline Astigmatismo miópico compuesto & 121 & 64.4 \\
\hline Astigmatismo mixto & 58 & 30.8 \\
\hline Astigmatismo miópico simple & 9 & 4.8 \\
\hline Miopía & 0 & 0 \\
\hline Hipermetropía & 0 & 0 \\
\hline Total (ojos) & $\mathbf{1 8 8}$ & $\mathbf{1 0 0}$ \\
\hline
\end{tabular}

Fuente: Historias clínicas.

Tabla 6. Distribución de los pacientes de acuerdo a patrones topográficos. Centro de Especialidades Médicas Fundación Donum, Cuenca, Ecuador (2015-2018)

\begin{tabular}{|l|c|c|}
\hline Patrones topográficos & Frecuencia & Porcentaje \\
\hline $\begin{array}{l}\text { Botón de camisa asimétrico } \\
\text { con desviación de ejes en J }\end{array}$ & 66 & 35.1 \\
\hline $\begin{array}{l}\text { Botón de camisa asimétrico } \\
\text { con encorvamiento inferior }\end{array}$ & 48 & 25.5 \\
\hline $\begin{array}{l}\text { Irregular } \\
\text { Botón de camisa asimétrico } \\
\text { con encorvamiento superior }\end{array}$ & 32 & 22.3 \\
\hline $\begin{array}{l}\text { Total (ojos) } \\
\text { Fuente: Historias clínicas. }\end{array}$ & $\mathbf{1 8 8}$ & 17.1 \\
\hline
\end{tabular}

Tabla 7. Distribución de la muestra según clasificación de Fernández-Vega Alfonso. Centro de especialidades médicas Fundación Donum, Cuenca, Ecuador (2015-2018)

\begin{tabular}{|l|c|c|}
\hline Típo de queratocono & Frecuencia & Porcentaje \\
\hline Fenotipo 2 & 48 & 25.5 \\
\hline Fenotipo 3 & 44 & 23.4 \\
\hline Fenotipo 1 & 36 & 19.2 \\
\hline Fenotipo 5 & 32 & 17.0 \\
\hline Fenotipo 4 & 28 & 14.9 \\
\hline Total (ojos) & $\mathbf{1 8 8}$ & $\mathbf{1 8 8}$ \\
\hline Fuente: Historias clínicas. & & \\
\hline
\end{tabular}

los 3 años de seguimiento. Además se obtuvo una disminución de casos con queratometrías menores a $45 \mathrm{D}$ a los 3 años del diagnóstico, con incremento de pacientes en los restantes grupos: 50-54.9, 55-59.9, 60 D o más.

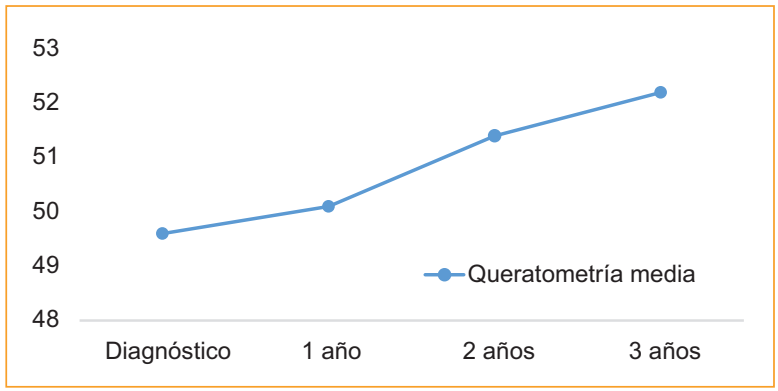

Figura 1. Queratometría media (promedio de la muestra).

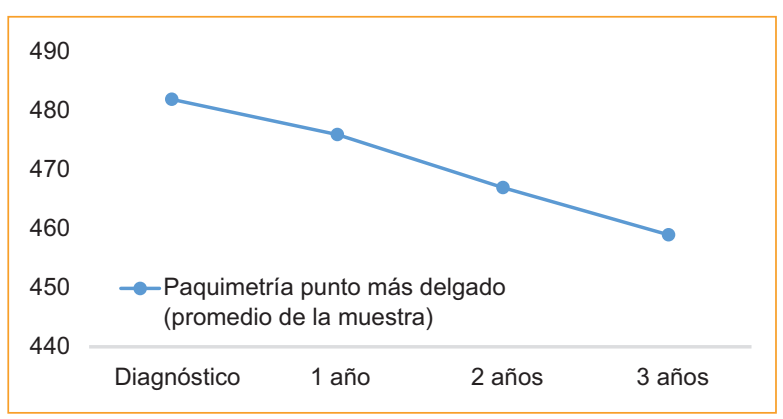

Figura 2. Paquimetría punto más delgado (promedio de la muestra).

Se obtuvo un aumento estadísticamente significativo entre el valor promedio de queratometría media de la muestra (49.6 D) en el momento del diagnóstico y el valor promedio de queratometría media a los 3 años de seguimiento (52.4 D). ( $p=0.02 ; \alpha=0.05$ ) (Fig. 1).

Como se ve en la tabla 9, en el momento del diagnóstico, hubo un predominio de casos con paquimetrías entre 480 y 499 um; sin embargo, a los 3 años de seguimiento se obtienen mayor cantidad de casos con paquimetrías entre 450 y $479 \mu \mathrm{m}$. Se obtuvo una disminución estadísticamente significativa entre la paquimetría promedio de la muestra en el momento del diagnóstico $(482 \mu \mathrm{m})$ y la paquimetría promedio de la muestra a los 3 años de seguimiento $(459 \mu \mathrm{m})(\mathrm{p}=0.03 ; \alpha=0.05)$ (Fig. 2).

La tabla 10 muestra un predominio de pacientes con cilindro refractivo entre 2 y $4.9 \mathrm{D}(41.0 \%)$ en el momento del diagnóstico. A los 3 años de seguimiento, este grupo se incrementa, así como los pacientes en los grupos de 5 a $8 \mathrm{D}$ y más de $8 \mathrm{D}$, a expensas de una disminución de los pacientes con menos de $2 \mathrm{D}$ de cilindro refractivo. Se obtuvo un incremento estadísticamente significativo entre el cilindro refractivo promedio de la muestra en el momento del diagnóstico (4.6 D) y el cilindro refractivo promedio de la muestra a los 3 años de seguimiento (5.1 D) $(p=0.01 ; \alpha=0.05)$ (Fig. 3). 
Tabla 8. Distribución de los pacientes de acuerdo a la queratometría media. Centro de Especialidades Médicas Fundación Donum, Cuenca, Ecuador (2015-2018)

\begin{tabular}{|l|c|c|c|c|}
\hline \multirow{2}{*}{ Queratometrías (dioptrías-D) } & \multicolumn{3}{|c|}{ Diagnóstico } & \multicolumn{2}{c|}{ 3 años } \\
\cline { 2 - 5 } & Frecuencia & Porcentaje & Frecuencia & Porcentaje \\
\hline Menor de 45 & 25 & 13.3 & 9 & 4.8 \\
\hline $45-49.9$ & 113 & 60.1 & 114 & 60.6 \\
\hline $50-54.9$ & 24 & 12.8 & 29 & 15.4 \\
\hline $55-59.9$ & 19 & 10.1 & 24 & 12.8 \\
\hline 60 o más & 7 & 3.7 & 12 & 6.4 \\
\hline Total (ojos) & $\mathbf{1 8 8}$ & $\mathbf{1 0 0}$ & $\mathbf{1 8 8}$ & $\mathbf{1 0 0}$ \\
\hline
\end{tabular}

$(\mathrm{p}=0.02 ; \alpha=0.05)$.

Al diagnóstico: Media 47.6; DE 0.67. A los 3 años: Media 48.1; DE: 0.32

Fuente: Historias clínicas.

Tabla 9. Distribución de los pacientes de acuerdo a la paquimetría (punto más delgado). Centro de Especialidades Médicas Fundación Donum, Cuenca, Ecuador (2015-2018)

\begin{tabular}{|c|c|c|c|c|}
\hline \multirow[t]{2}{*}{ Paquimetría (pm) } & \multicolumn{2}{|c|}{ Diagnóstico } & \multicolumn{2}{|c|}{3 años } \\
\hline & Frecuencia & Porcentaje & Frecuencia & Porcentaje \\
\hline Mayor a 500 & 12 & 6.4 & 7 & 3.7 \\
\hline $480-499$ & 73 & 38.8 & 57 & 30.4 \\
\hline $450-479$ & 55 & 29.3 & 66 & 35.1 \\
\hline $400-449$ & 39 & 20.7 & 45 & 23.9 \\
\hline Menor de 400 & 9 & 4.8 & 13 & 6.9 \\
\hline Total (ojos) & 188 & 100 & 188 & 100 \\
\hline
\end{tabular}

$(p=0.03 ; \alpha=0.05)$.

Al diagnóstico: Media 477.2; DE 0.27. A los 3 años: Media 468.1; DE 0.52.

Fuente: Historias clínicas.

Tabla 10. Distribución de los pacientes de acuerdo al cilindro refractivo. Centro de Especialidades Médicas Fundación Donum, Cuenca, Ecuador (2015-2018)

\begin{tabular}{|c|c|c|c|c|}
\hline \multirow{2}{*}{$\begin{array}{l}\text { Cilindro } \\
\text { refractivo }\end{array}$} & \multicolumn{2}{|c|}{ Diagnóstico } & \multicolumn{2}{|c|}{3 años } \\
\hline & Frecuencia & Porcentaje & Frecuencia & Porcentaje \\
\hline Menor 2.0 & 29 & 15.4 & 13 & 6.9 \\
\hline $2-4.9$ & 77 & 41.0 & 84 & 44.7 \\
\hline $5-8$ & 56 & 29.8 & 62 & 33.0 \\
\hline Más de 8 & 26 & 13.8 & 29 & 15.4 \\
\hline Total (ojos) & 188 & 100 & 188 & 100 \\
\hline
\end{tabular}

En la tabla 11 vemos un aumento de casos con agudeza visual inferior a 20/400 a los 3 años de seguimiento, con predominio en ambos momentos del estudio de pacientes en el grupo con agudeza visual entre 20/400 y 20/100, además, se observa disminución de casos con agudeza visual sin corrección mayor a 20/100.

La tabla 12 muestra que existe una disminución de casos con agudeza visual con corrección superior a 20/40 a los 3 años de seguimiento. Se observa, además, un aumento de casos con agudeza visual entre 20/100 y 20/400 y mayor frecuencia de casos con menos de 20/400, en comparación con el momento del diagnóstico. 
Tabla 11. Distribución de los pacientes de acuerdo a la agudeza visual sin corrección. Centro de Especialidades Médicas Fundación Donum, Cuenca, Ecuador (2015-2018)

\begin{tabular}{|l|c|c|c|c|}
\hline \multirow{2}{*}{ Agudeza visual sin corrección } & \multicolumn{3}{|c|}{ Diagnóstico } & \multicolumn{2}{c|}{3 años } \\
\hline & Frecuencia & Porcentaje & Frecuencia & Porcentaje \\
\hline Menor a $20 / 400$ & 23 & 12.2 & 29 & 15.4 \\
\hline Entre 20/400 y 20/100 & 117 & 62.3 & 128 & 68.1 \\
\hline Mayor a 20/100 y menor a 20/40 & 42 & 22.3 & 29 & 15.4 \\
\hline Entre 20/40 y 20/20 & 6 & 3.2 & 2 & 1.1 \\
\hline Total (ojos) & $\mathbf{1 8 8}$ & $\mathbf{1 0 0}$ & $\mathbf{1 8 8}$ & $\mathbf{1 0 0}$ \\
\hline
\end{tabular}

Tabla 12. Distribución de los pacientes de acuerdo a la agudeza visual con corrección. Centro de Especialidades Médicas Fundación Donum, Cuenca, Ecuador (2015-2018)

\begin{tabular}{|l|c|c|c|c|}
\hline \multirow{2}{*}{ Agudeza visual con corrección } & \multicolumn{3}{|c|}{ Diagnóstico } & \multicolumn{2}{c|}{3 años } \\
\hline Menor a 20/400 & Frecuencia & Porcentaje & Frecuencia & Porcentaje \\
\hline Entre 20/400 y 20/100 & 7 & 3.7 & 11 & 5.9 \\
\hline Mayor a 20/100 y menor a 20/40 & 77 & 41.0 & 98 & 52.1 \\
\hline Entre 20/40 y 20/20 & 68 & 36.2 & 57 & 30.3 \\
\hline Total (ojos) & 36 & 19.1 & 22 & 11.7 \\
\hline
\end{tabular}

Fuente: Historias clínicas.

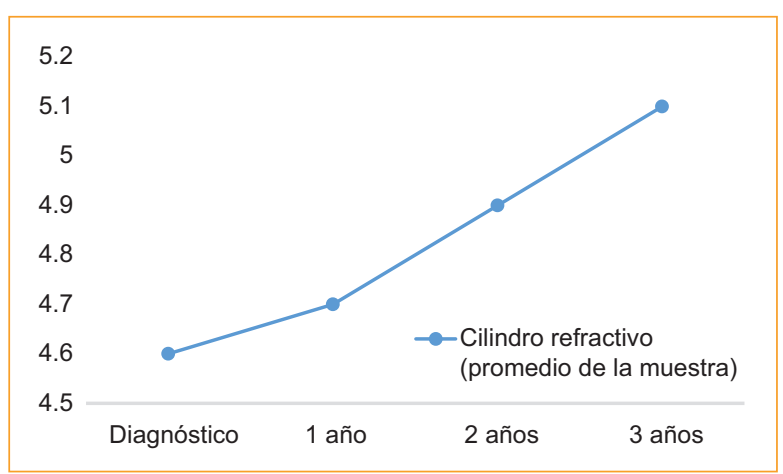

Figura 3. Cilindro refractivo (promedio de la muestra).

\section{Discusión}

El KC es una enfermedad de pacientes jóvenes ${ }^{12-14}$. Suele aparecer en la segunda década de la vida, aunque se han reportado casos de menor edad ${ }^{15-18}$. Por ello, el interés del actual estudio en destacar la presencia de la enfermedad desde la infancia, coincidimos con estudios realizados del tema que estudian casos de $\mathrm{KC}$ con presentación en edad pediátrica ${ }^{19-23}$. En el caso de nuestro estudio son pacientes que habitualmente llegaron a la consulta por diferentes motivos: examen oftalmológico para entrada a instituciones escolares, renovación de la corrección óptica, antecedentes familiares de la enfermedad e interés de los padres en un estudio topográfico corneal, entre otros.

En cuanto al sexo, no existe consenso en la literatura con respecto a si la enfermedad tiene mayor frecuencia en uno u otro, diferentes series reportan la enfermedad en ambos sexos, sin existencia de diferencias estadísticamente significativas entre ellos ${ }^{12,19,20}$. La enfermedad tiene carácter hereditario y existen familias donde tanto hijos varones como féminas heredan la enfermedad 22-24.

Pérez ${ }^{25}$ obtiene que el $59.7 \%$ de los pacientes correspondió al sexo femenino; la edad media fue de 35 años, y por grupo de edades, el intervalo de 30 a 39 años fue el más representado porcentualmente, con promedios de edades por sexo más o menos similares. 
Marrero ${ }^{12}$ obtiene que el grupo etario más afectado fue el de 30 a 39 años (52.9\%), seguido en orden de frecuencia por el de 20 a 29 (35.2\%); mientras que, en relación con el sexo, predominaron las féminas (58.8\%). Refik $^{13}$ muestra una media de edad de 25 años sin diferencias significativas en cuanto al sexo.

Respecto al lugar de procedencia de los pacientes del estudio, por razones geográficas, la mayoría de los pacientes son provenientes de Cuenca, lugar en que se encuentra la institución donde se realizó la investigación, seguido por regiones cercanas a la ciudad en las que no hay centros con la tecnología necesaria para el diagnóstico del KC por Pentacam. Estos pacientes pertenecientes a otras regiones, incluso otras provincias del país, vienen referidos por médicos de atención primaria o por oftalmólogos generales, con el objetivo de una valoración subespecializada y la personalización del tratamiento.

En cuanto a enfermedades asociadas, los resultados obtenidos son similares a los estudios existentes de la enfermedad. Pérez ${ }^{25}$ reporta que el $26.4 \%$ presentaba alguna enfermedad, en correspondencia mayormente con el asma bronquial y las alergias (13.9\% y $12.5 \%$, respectivamente). La queratoconjuntivitis alérgica (21.9\%) fue la afección oftalmológica más frecuente.

Referente a los síntomas y signos encontrados, se coincide con otros estudios como Pérez ${ }^{25}$, que obtiene que la mitad de los enfermos refirieron disminución lenta y progresiva de la visión, el $34.7 \%$ cambiaron frecuentemente de corrección óptica. Los síntomas como prurito e intolerancia al lente de contacto fueron descritos por el $18.1 \%$ de los pacientes. Pérez ${ }^{25}$ obtiene como signos más frecuentes la córnea cónica (38.3\%), signo de Munson (33.6\%), nervios corneales prominentes $(25.8 \%)$ y el signo de tijera por esquiascopia (65.6\%).

Los defectos refractivos relacionados con la enfermedad e incluidos en el cuadro clínico de la misma son la miopía y el astigmatismo, en su forma más frecuente se presentan como astigmatismos miópicos, en su mayoría de tipo compuesto. La miopía se debe a una mayor curvatura corneal, dada la forma cónica que adquiere la córnea, con curvaturas que superan las 43 $\mathrm{D}$, lo que aumenta el índice de refracción ocular y el punto focal se forma delante de la retina. La presencia de astigmatismo se debe a una distorsión o alteración de los meridianos corneales, en su curvatura anterior y/o posterior corneal, lo cual induce presencia de aberraciones ópticas, sobre todo en astigmatismos de tipo irregular.
El actual estudio coindice con resultados internacionales del tema, donde estos defectos refractivos son los más frecuentes ${ }^{26-29}$. Pérez ${ }^{25}$ obtiene que el astigmatismo miópico compuesto se presentó significativamente $(p=0.000)$ en el $85.2 \%$ de los ojos, mientras que las restantes formas de astigmatismo fueron infrecuentes, por debajo del $10 \%$.

No obstante, se debe destacar la presencia en segundo lugar por orden de frecuencia de astigmatismos mixtos, por lo que la esfera de la refracción es positiva. Son casos que no se han reportado en la literatura y fueron encontrados en el actual estudio. La diferencia del componente esférico de la refracción depende de la ubicación del cono, existen KC de diferente ubicación corneal, y esto puede inducir variaciones en la esfera de la refracción.

Los patrones de topografía corneal obtenidos evidencian una correspondencia con los defectos refractivos hallados. La presencia de patrones en botón de camisa asimétrico con desviación del eje en $\mathrm{J}$ de astigmatismos regulares asimétricos inferiores refleja que la mayoría de las ubicaciones del cono son inferiores; no obstante, se obtuvieron casos con asimetrías superiores y patrones de ectasia corneal con presencia de astigmatismos irregulares.

La topografía corneal continúa siendo un pilar fundamental en el diagnóstico de la enfermedad, por la integralidad de elementos que aporta, incluidos paquimetría, queratometría, mapas, índices de curvaturas corneales anterior y posterior, así como la posibilidad de realizar un análisis integrado de coincidencia o proximidad de sitios más curvos con zonas de mayor adelgazamiento. En el estudio de Pérez ${ }^{25}$, según el patrón topográfico, el astigmatismo irregular fue significativamente superior $(p=0.000)$, con frecuencia alrededor del $99.2 \%$.

El advenimiento y desarrollo de los topógrafos corneales ha posibilitado el diagnóstico precoz de la enfermedad, es por ello que en la muestra de estudio obtenemos casos con KC en los primeros estadios, donde aún no se evidencian patrones topográficos de ectasia corneal evidentes; sin embargo, el análisis integral de la topografía más los signos y síntomas posibilita el diagnóstico ${ }^{30-33}$.

La existencia de varios topógrafos (Pentacam) en nuestro medio ha posibilitado la accesibilidad a este estudio, por lo que se examinan con mayor profundidad pacientes con defectos refractivos miópicos y astigmáticos, donde se puede sospechar la enfermedad en sus primeros estadios, o la presencia de KC frustres, con la posibilidad de seguimiento del paciente a largo plazo 


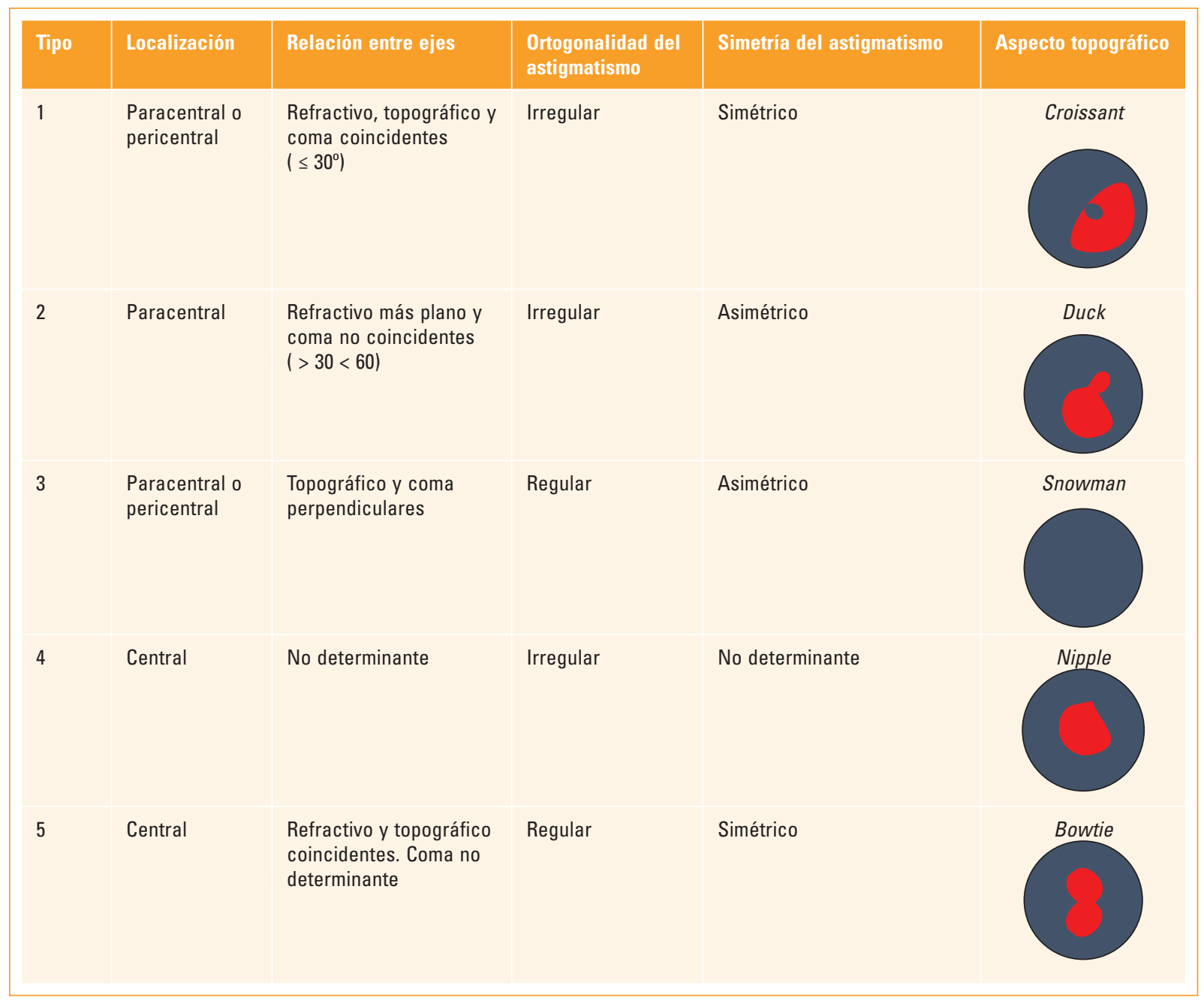

Figura 4. Clasificación Fernández-Vega A. del Queratocono.

y la comparación de la primera topografía con las siguientes, en vistas a corroborar la aparición de la enfermedad o su evolución o no, aspecto que adquiere mayor relevancia en pacientes en edad pediátrica, en que es necesario un diagnóstico precoz ${ }^{34}$.

La clasificación de Fernández-Vega es un análisis desde la perspectiva morfológica del cono, dirigida fundamentalmente al tratamiento con anillos intraestroma$\operatorname{les}^{35,36}$. En este sentido, incluye aspectos como: localización del cono, relación entre los 3 ejes diagnósticos principales (refractivo más plano, topográfico más plano y coma), ortogonalidad, simetría y aspecto topográfico del mapa de curvatura. En edad pediátrica obtuvimos predominio de KC de fenotipo 2, que se caracteriza por su ubicación paracentral, con eje topográfico más plano, no coincidente con eje comático, con ectasia localizada a más de $0.6 \mathrm{~mm}$ del centro pupilar y aspecto topográfico en mapa tangencial de patrón en «pajarita» con lóbulos asimétricos y angulados entre sí, con imagen en «pato» (duck). Además, se obtuvo predominio del fenotipo 3 también de ubicación parecentral con aspecto topográfico de imagen en «muñeco de nieve» (snowman). En este fenotipo existe perpendicularidad entre los ejes y se definen dos subtipos. La posibilidad de que estos patrones morfológicos tengan variaciones en el futuro es muy probable, teniendo en cuenta la modificación de los ejes refractivo, topográfico y comático, para ello se necesitan estudios a más largo plazo que evalúen estas modificaciones (Fig. 4).

Los valores de queratometría obtenidos en el estudio evidencian el aumento de la curvatura corneal en la enfermedad, resultados similares a los obtenidos anteriormente por diferentes autores, donde todos coinciden en el incremento de las queratometrías en el KC, que se explica por los aspectos fisiopatológicos del $\mathrm{KC}^{37-38}$. En cuanto al poder queratométrico de la 
córnea central, Pérez ${ }^{25}$ reporta que los tres grupos queratométricos siguen igual distribución. Refik ${ }^{13}$ obtiene una disminución de la queratometría media, cercana a los 6 meses de cross linking. El aumento significativo de la queratometría media en el grupo de estudio expone la evolución de la enfermedad en esta edad, en comparación con otros grupos de edades no pediátricas.

En cuanto a los valores paquimétricos, en la enfermedad se produce un adelgazamiento de la córnea, por lo que la mayoría de los pacientes se encuentran en rangos inferiores a las $500 \mu \mathrm{m}$. Los resultados obtenidos coinciden con otras series de estudios, que reflejan pacientes entre las 400 y $500 \mu \mathrm{m}$. En el estudio de Pérez ${ }^{25}$, el espesor medio de la córnea central fue de $493.2 \mu \mathrm{m}$, con valores que oscilaron entre $485.6 \mathrm{y}$ $500.8 \mu \mathrm{m}$. Refik ${ }^{13}$ obtiene una disminución de la paquimetría corneal central con respecto a niveles preoperatorios a los 6 meses de cross linking. La existencia de un grupo de pacientes con paquimetrías cercanas a las $500 \mu \mathrm{m}$ refleja el diagnóstico en estadios iniciales de la enfermedad. La disminución significativa de la paquimetría en el punto más delgado, obtenida en la muestra, constituye un factor pronóstico importante, analizada en conjunto con variables como la edad del paciente, el cilindro refractivo y la queratometría en el punto más curvo. Además, tiene gran validez en el análisis la correspondencia entre el sitio de queratometría más curva y el punto corneal más delgado.

El KC se caracteriza por la presencia de astigmatismos irregulares, por lo que es de esperar en la muestra estudiada cilindros refractivos elevados, incluso hasta $8 \mathrm{D}$, lo cual es un resultado similar al obtenido por otros autores $^{39-41}$. La evolución del cilindro refractivo, con incremento durante los 3 años de estudio, es la expresión de las modificaciones en la curvatura corneal ya descritas.

Marrero ${ }^{12}$ obtiene primacía de la agudeza visual sin corrección por debajo de 0.1 (47.0\%), y en el $55.8 \%$ se alcanzó una agudeza visual entre 0.3 y la unidad de visión con corrección óptica, seguida en menor cuantía por el $32.3 \%$ de los estudiados por debajo de 0.3 y 0.1 . Pére $z^{25}$ obtiene que la media de la agudeza visual sin corrección fue de 0.1 , en tanto en la agudeza visual con corrección fue de $0.6 \pm 0.3$ líneas, donde se observó un aumento significativo de la agudeza visual con corrección. Refik ${ }^{13}$ reporta una mejoría significativa de la agudeza visual con corrección a los 6 meses de cross linking.

El aspecto más significativo en el análisis y discusión de los resultados obtenidos, sin duda, es la evolución de la enfermedad. La forma de evaluar este aspecto incluye los valores de paquimetría, queratometría, cilindro refractivo en la topografía corneal. En el actual estudio fueron reevaluadas las variables a los 3 años, con diferencias estadísticamente significativas en las modificaciones de dichas variables, lo cual reafirma el concepto de la evolución de la ectasia desde edades tempranas, aspecto que se evidencia aún más por las diferencias de agudeza visual en el diagnóstico y a los 3 años de seguimiento.

Aunque el KC se diagnostica con mayor frecuencia después de la adolescencia, el proceso de ectasia corneal comienza a una edad mucho más temprana ${ }^{42}$. El KC pediátrico (KC que se manifiesta en la infancia [menores de 18 años] o la adolescencia [entre los 10 y los 19 años]) presenta varias características únicas. Los estudios han demostrado que el KC pediátrico muestra una tasa más alta ( $88 \%$ de los ojos queratocónicos) y mayor velocidad de progresión del KC en comparación con el KC adulto $20,39,43,44$. Léoni-Mesplié obtuvo que el KC en los niños fue significativamente más grave en el momento del diagnóstico, con un $27.8 \%$ en etapa 4 frente a un $7.8 \%$ en el caso de los adultos, y el KC evolucionó más rápido en los niños en comparación con el grupo de adultos ${ }^{20}$. Además, la rigidez biomecánica de la córnea está inversamente relacionada con la edad, y los niños con queratoconjuntivitis vernal coexistente son mucho más susceptibles. Por lo tanto, la progresión del KC en los niños es agresiva y puede que no se detenga por sí sola $26,37,40$. Esto puede llevar a un deterioro visual progresivo en los pacientes pediátricos y afectar su desarrollo social y educativo y, por lo tanto, afectar negativamente a su calidad de vida ${ }^{45,46}$.

La presencia de astigmatismos por encima de 2 dioptrías en niños con o sin alergia ocular deben constituir criterios de tamizaje directo por Pentacam, y según los resultados, debe hacerse seguimiento cada 6 meses o anual con topografía. La presencia de patrones topográficos de astigmatismo regulares, ortogonales, con ligeras asimetrías superiores o inferiores deben hacer pensar en seguimiento del caso, más aún si ya se acompaña de valores sospechosos de algunos índices relacionados con la enfermedad y paquimetrías cercanas a las $500 \mu \mathrm{m}$. No se debe esperar a la presencia de un diagnóstico con todos los índices topográficos y a valores inferiores a $480 \mu \mathrm{m}$ de paquimetría para iniciar el tratamiento.

Es deber del oftalmólogo explicar a padres y/o tutores la importancia del estudio de sus hijos desde 
edades tempranas, máxime si existe antecedente familiar de KC. Son aspectos que resultan evidentes para la comunidad médica de la especialidad, sin embargo, deben explicarse insistentemente.

\section{Conclusiones}

- El KC en edad pediátrica fue más frecuente en pacientes adolescentes, con edades comprendidas entre los 14 y 18 años, del sexo masculino provenientes en su mayoría de la ciudad de Cuenca.

- Los pacientes en su gran mayoría presentaban queratoconjuntivitis alérgicas, alergia sistémica y dermatitis atópica, consultaron por disminución de la agudeza visual y prurito con presencia de signo en tijera, signo de Munson y nervios corneales prominentes.

- Los defectos refractivos predominantes fueron el astigmatismo miópico compuesto y el astigmatismo mixto con patrones topográficos en botón de camisa asimétrico con desviación de ejes en $\mathrm{J}$ y botón de camisa asimétrico con encorvamiento inferior. Se identificaron con mayor frecuencia los fenotipos 2 y 3 de la enfermedad.

- La agudeza visual se mantuvo con predominio de pacientes entre $20 / 100$ y 20/400, que mostraron modificaciones de queratometría, cilindro refractivo y paquimetría, que sugieren progresión de la enfermedad en este grupo de edad, en un periodo de 3 años.

\section{Conflicto de intereses}

Los autores declaran que no existen conflictos de intereses.

\section{Financiamiento}

Los autores declaran que no han recibido financiación para la realización de la investigación.

\section{Responsabilidades éticas}

Protección de personas y animales. Los autores declaran que los procedimientos seguidos se conformaron a las normas éticas del comité de experimentación humana responsable y de acuerdo con la Asociación Médica Mundial y la Declaración de Helsinki.
Confidencialidad de los datos. Los autores declaran que han seguido los protocolos de su centro de trabajo sobre la publicación de datos de pacientes.

Derecho a la privacidad y consentimiento informado. Los autores han obtenido el consentimiento informado de los pacientes y/o sujetos referidos en el artículo. Este documento obra en poder del autor de correspondencia.

\section{Bibliografía}

1. Kankariya VP, Kymionis GD, Diakonis VF, Yoo SH. Management of pediatric keratoconus - Evolving role of corneal collagen cross-linking: An update. Indian J Ophthalmol. 2013;61(8):435-40.

2. Galvis V, Sherwin T, Tello A, Merayo J, Barrera R, Acera A. Keratoconus: an inflammatory disorder? Eye. 2015;29:843-59.

3. Konstantopoulos A, Mehta JS. Conventional versus accelerated collagen cross- linking for keratoconus. Eye Contact Lens. 2015;41(2):65-7.

4. Mastropasqua L. Collagen cross-linking: When and how? A review of the state of the art of the technique and new perspectives. Eye Vis (Lond). 2015;2:19.

5. Soiberman U, Foster JW, Jun AS, Chakravarti S. Pathophysiology of Keratoconus: What Do We Know Today. Open Ophthalmol J. 2017; 11:252-61.

6. Hammer A, Richoz O, Arba Mosquera S, Tabibian D, Hoogewoud F, Hafezi F. Corneal biomechanical properties at different corneal cross-linking (CXL) irradiances. Invest Ophthalmol Vis Sci. 2014;55(5):2881-4.

7. Soeters N, van der Valk R, Tahzib NG. Corneal cross-linking for treatment of progressive keratoconus in various age groups. J Refract Surg. 2014; 30(7):454-60.

8. El Rami H, Chelala E, Dirani A, Fadlallah A, Fakhoury H, Cherfan C, et al. An Update on the Safety and Efficacy of Corneal Collagen Cross-Linking in PediatricKeratoconus. Biomed Res Int. 2015;2015:257-927.

9. Abril LE. Estudio topográfico de pacientes diagnosticados con queratocono en la costa y sierra ecuatoriana en el periodo agosto 2014 a enero 2015. Tesis. Instituto Tecnológico Cordillera. Ecuador. 2015.

10. Mansfield N. El queratocono en pacientes de una institución privada de la ciudad de Quito, en el periodo de enero de 2015 a octubre de 2016. Tesis. Universidad de los Andes. Ecuador. 2017.

11. Reynoso MF, Castañeda GF. Diagnóstico y características del queratocono por topografía corneal en el Hospital Santa Inés Cuenca, 2012-2014 [tesis]. Universidad de Cuenca, Ecuador; 2016.

12. Marrero E, Sánchez O, Barrera BR, Díaz S, Somoza JA. Caracterización de pacientes con queratocono. Rev MEDISAN. 2011;15(12):1698-704.

13. Refik O, Gunhal S, Meryem D, Mehmet K, Hurkan K, Mehmet O, et al Six-month outcomes of corneal crosslinking with dextran-free isotonic riboflavin solution. Arq Bras Oftalmol. 2016;79(3):147-50.

14. Kaya V, Utine CA, Yılmaz ÖF. Intraoperative corneal thickness measurements during corneal collagen cross-linking with hypoosmolar riboflavin solution in thin corneas. Cornea. 2012;31(5):486-90.

15. Vinciguerra $P$, Randleman JB, Romano V, Legrottaglie EF, Rosetta $P$ Camesasca FI, Piscopo R, Azzolini C, Vinciguerra R. Transepithelial iontophoresis corneal collagen cross-linking for progressive keratoconus: initial clinical outcomes. J Refract Sur. 2014;30(11):746-53.

16. Buzzonetti L, Petrocelli G, Valente P, larossi G, Ardia R, Petroni S lontophoretic transepithelial corneal cross-linking to halt keratoconus in pediatric cases: 15-month follow-up. Cornea. 2015;34(5):512-5.

17. Magli A, Chiariello Vecchio E, Carelli R, Piozzi E, Di Landro F, Troisi S Pediatric keratoconus and iontophoretic corneal crosslinking: refractive and topographic evidence in patients underwent general and topical anesthesia, 18 months of follow-up. Int Ophthalmol. 2016;36(4):585-90.

18. Wollensak G, Spoerl E, Seiler T. Riboflavin/ultraviolet-a-reduced collagen crosslinking for the treatment of keratoconus. Am J Ophthalmol. 2003;135(5):620-7.

19. Jian $W$, Shen $Y$, Chen $Y$, Tian M, Zhou $X$. Ocular dimensions of the Chinese adolescents with keratoconus. BMC Ophthalmol. 2018;18(1):43.

20. Léoni-Mesplié S, Mortemousque B, Touboul D, Malet F, Praud D, Mesplié N, et al. Scalability and severity of keratoconus in children. Am J Ophthalmol. 2012;154:56-62.

21. Ucakhan OO, Bayraktutar BN, Saglik A. Pediatric corneal collagen cross-linking: long-term follow-up of visual, refractive, and topographic outcomes. Cornea. 2016;35:162-8.

22. Kankariya VP, Kymionis GD, Diakonis VF, Yoo SH. Management of pediatric keratoconus-evolving role of corneal collagen cross-linking: an update. Indian J Ophthalmol. 2013;61(8):435-40.

23. Godefrooij DA, de Wit GA, Uiterwaal CS, Imhof SM, Wisse RPL. Age-specific incidence and prevalence of keratoconus: a nationwide registration study. American Journal of Ophthalmology. 2017;175:169-72. 
24. Agrawal V. Long-term results of cornea collagen cross-linking with riboflavin for keratoconus. Indian J Ophthalmol. 2013;61(8):433-4.

25. Pérez Z, Ulloa S, Escalona E, Castillo A, Márquez S. Caracterización clínica y epidemiológica del queratocono. Rev Cubana Oftalmol. 2014;27(4).

26. Ertan A, Muftuoglu $O$. Keratoconus clinical findings according to differen age and gender groups. Cornea. 2008;27:1109-13.

27. Kamiya K, Shimizu K, Igarashi A, Miyake T. Assessment of anterior, posterior, and total central corneal astigmatism in eyes with keratoconus. Am J Ophthal. 2015;160(5):851-7.

28. Feizi S, Delfazayebaher S, Javadi MA, Karimian F, Ownagh V, Sadeghpour F. Mean Posterior Corneal Power and Astigmatism in Normal versus Keratoconic Eyes. J Ophthalmic Vis Res. 2018;13(2):93-100.

29. Shajari M, Friderich S, Pour Sadeghian M, Schmack I, Kohnen T. Characteristics of Corneal Astigmatism of Anterior and Posterior Surface in a Normal Control Group and Patients With Keratoconus. Cornea. 2017; 36:457-62.

30. Savini G, Næser K, Schiano-Lomoriello D, Mularoni A. Influence of Posterior Corneal Astigmatism on Total Corneal Astigmatism in Eyes With Keratoconus. Cornea. 2016;35:1427-33.

31. Naderan M, Rajabi MT, Zarrinbakhsh P. Distribution of Anterior and Posterior Corneal Astigmatism in Eyes With Keratoconus. Am J Ophthalmol. 2016;167:79-87.

32. Reddy JC, Rapuano CJ, Cater JR, Suri K, Nagra PK, Hammersmith KM Comparative evaluation of dual Scheimpflug imaging parameters in keratoconus, early keratoconus, and normal eyes. J Cataract Refract Surg. 2014;40:582-92.

33. Kamiya K, Ishii R, Shimizu K, Igarashi A. Evaluation of corneal elevation, pachymetry and keratometry in keratoconic eyes with respect to the stage of Amsler-Krumeich classification. Br J Ophthalmol. 2014;98:459-63.

34. Bernal N, Arias A, Ortega L, Cuevas J. Utilidad de la tomografía cornea pentacam en el queratocono en niños. Oftalmol Clin Exp. 2011:5(1):18-27.

35. Alfonso JF, Lisa C, Fernández-Vega Cueto L, Poo A, Madrid D. Clasificación del queratocono basada en fenotipos clínicos. Influencia del as- tigmatismo congénito en la morfología del queratocono. Biomecánica y arquitectura corneal. 2014;165-84.

36. Lisa Fernández C. Corrección del queratocono con segmentos intracorneales tipo Ferrara: estrategia personalizada para su implante [tesis doctoral]. Universidad de Oviedo; 2017.

37. Kamiya K, Shimizu K, Ohmoto F. Effect of aging on corneal biomechanical parameters using the ocular response analyzer. J Refract Surg. 2009;25:888-93.

38. Takahashi M, Kamiya K, Kono Y, Shoji N. Time Course of Changes in Simulated Keratometry and Total Corneal Refractive Power after Corneal Collagen Cross-Linking for Progressive Keratoconus. Biomed Res Int. 2018;2620784.

39. Li X, Yang H, Rabinowitz YS. Longitudinal study of keratoconus progression. Exp Eye Res. 2007;85:502-7.

40. Reeves SW, Stinnett S, Adelman RA, Afshari NA. Risk factors for progression to penetrating keratoplasty in patients with keratoconus. Am J Ophthalmol. 2005;140:607-11.

41. Plazzi F, Amaral A, Gambi L, Pinheiro L, Amaral B. Corneal collagen cross-linking for progressive keratoconus in patients aged 9 to 14 . Rev Bras Oftalmol. 2016;75(2):84-8

42. Rabinowitz YS. Keratoconus. Surv Ophthalmol. 1998;42:297-319.

43. Chatzis N, Hafezi F. Progression of keratoconus and efficacy of pediatric [corrected] corneal collagen cross-linking in children and adolescents. J Refract Surg. 2012;28:753-8.

44. Al Suhaibani AH, Al-Rajhi AA, Al-Motowa S, Wagoner MD. Inverse relationship between age and severity and sequelae of acute corneal hydrops associated with keratoconus. Br J Ophthalmol. 2007;91:984-5.

45. Nasrin F, lyer RV, Mathews SM. Simultaneous Estimation of Corneal Topography, Pachymetry, and Curvature. IEEE Trans Med Imaging. 2018;37(11):2463-73.

46. Or L, Rozenberg A, Abulafia A, Avni I, Zadok D. Corneal Cross-Linking in Pediatric Patients: Evaluating Treated and Untreated Eyes-5-Year Follow-Up Results. Cornea. 2018;37(8):1013-7. 